\title{
Pontocerebellar hypoplasia type 2
}

INSERM

\section{Source}

INSERM. (1999). Orphanet: an online rare disease and orphan drug data base.

Pontocerebellar hypoplasia type 2. ORPHA:2524

Pontocerebellar hypoplasia type $2(\mathrm{PCH} 2)$ is the most common subtype of

pontocerebellar hypoplasia (see this term) characterized by neonatal onset and a lack of voluntary motor development and later progressive microencephaly, generalized clonus, development of chorea and spasticity. The majority of patients will not reach puberty. 